\title{
Investigation and Countermeasures of Psychological Status of Outpatient Guide Nurses During the Outbreak of COVID-19 Pandemic
}

\author{
Meihua Zhong ${ }^{1}$, Lin Wang ${ }^{2,}$, Xiaojin Liu ${ }^{1}$, Jinghui Zhang ${ }^{1}$ \\ ${ }^{1}$ Department of Outpatient, the First Affiliated Hospital of Jinan University, Guangzhou, China \\ ${ }^{2}$ Department of Transitional Care, the First Affiliated Hospital of Jinan University, Guangzhou, China \\ Email address: \\ zmh666jnu@126.com (Meihua Zhong),wanglin18903078509@126.com (Lin Wang) \\ ${ }^{*}$ Corresponding author
}

To cite this article:

Meihua Zhong, Lin Wang, Xiaojin Liu, Jinghui Zhang. Investigation and Countermeasures of Psychological Status of Outpatient Guide Nurses During the Outbreak of COVID-19 Pandemic. American Journal of Nursing Science. Vol. 9, No. 4, 2020, pp. 186-189.

doi: 10.11648/j.ajns.20200904.11

Received: April 28, 2020; Accepted: May 18, 2020; Published: May 29, 2020

\begin{abstract}
Objective Investigation and countermeasures of psychological status of outpatient guide nurses under epidemic situation of COVID-19. Methods The group focus interview method was used to collect data onto 9 outpatient guide nurses in our hospital, the content of the data mainly focuses on the psychological feelings of the guide nurses under the epidemic situation, colorize analysis method was used to analyze the interview data. Results In the case of COVID-19, the guide nurses quickly adapted to the current situation, actively served each patient their families, and did well in preview and triage, consultation, and epidemic prevention and control education and so on, after they had experienced worry, fear, anxiety, nervousness, reluctance to go to work, and the contradiction and hesitation in triage. Conclusion COVID-19 outbreak, the mental health status of outpatient guide nurses needs to pay close attention to, to do a good job of the work process guidelines on COVID-19, strengthen protection knowledge and skills training, ensure that the protective supplies are adequate, give humanistic care and offer psychological intervention, to provide a powerful psychological support for the nurses in the triage work, so as to contribute to the epidemic prevention and control of hospitals and countries.
\end{abstract}

Keywords: COVID-19, Guide Nurse, Psychological Status

\section{Introduction}

In early 2020, a new corona-virus pneumonia (short for NCP) [1] was blown out at the first place in Wuhan, and then the epidemic spread throughout the country. In response to the outbreak, Guangdong Province launched a 1-level response to major public health incidents [2], the major hospitals in Guangzhou quickly set up fever per-testing triage and fever clinics. The service desk nurses in our hospital are responsible for outpatient triage consultation, therefore, they are required to measure body temperature and provide triage consultation services for patients and their families in close distance, Improper protection during the contact process may cause NCP infection, which will have a serious impact on the psychology of the help desk nurses. In response to this problem, the author used the qualitative interview video conference group focus interview method to interview the nurses in charge of our hospital's desk, and found that the nurses showed negative emotions such as worry, fear, tension and anxiety, and there was obvious psychological stress The response is consistent with the psychological survey results of the psychological investigation of first-line nurses during the period of SARS, such as Chaos Weiss, which provides a basis for the follow-up psychological intervention, which can better take corresponding countermeasures and alleviate bad emotions. The research shows that, in addition to having a high degree of responsibility, professional ethics and professional medical knowledge, the nurses at the outpatient service desk also need to overcome their own worry, fear, anxiety and tension; While they do a good job of personal safety protection, they also need to provides the triage service with professional, careful and patient for the patients' families, 
"well checking", "well triage" and "well stand guard", to make a contribution to the epidemic prevention and control of the hospital.

\section{Objects and Methods}

\subsection{Normal Data}

The cluster sampling method was used to interview the nurses engaged in the outpatient service desk in our hospital. The interviewee inclusion criteria: engaged in the outpatient service desk for more than half a year; willing to participate in this research. A total of 9 nurses were interviewed, all females, the range of their age is $39-54$ years, average age ( $47 \pm 5.0)$ years; the years of the outpatient service desk work is $3-12$ years, average service desk working years $(6.4 \pm 2.7)$ years; including 1 head nurse, 8 nurses; 6 undergraduates, 3 junior colleges; 6 supervisor nurses and 3 senior nurses.

\subsection{Research Methods}

In the form of the group focus interview method [3] video special topic meeting, the host will first explain the purpose and method of this interview, and then guide the interviewees to participate in the discussion of the journey of the heart of the outbreak after obtaining the consent to them. The discussion venue is quiet and without interference. Try not to interrupt the participants during the interview and confirm their opinions timely. The interviewer will record the content of the interview and record. The interview time is about 1 hour based on the principle of saturated information.

\subsection{Data Collation and Analysis}

Within 24 hours after the interview, the recorded materials were transcribed verbatim and double-checked to ensure the accuracy of the data. Using Colorize seven-step analysis method [4] to analyze the data. (1) Read all records carefully; (2) extract statements had great significance; (3) summarize and extract significance from meaningful statements; (4) find common concepts or features of the significance form topics and topic groups; (5) connect topics with research phenomena to complete the narrative; (6) state the essential structure that constitutes this phenomenon; (7) return to the participants for verification. Two researchers will analyze the same data continuously and repeatedly, and compared it with the original sentence continuously; if there were inconsistencies, they would discuss and unify with the third party. Finally, return the different descriptions formed into the research object to check the authenticity of the data.

\section{Result}

According to the content of the interview, four themes can be drawn: worry, fear, nervousness, anxiety [5]. From its evolution, there have been four stages changes of work attitude.

\subsection{The Fear of Being Infected in Epidemic Situation}

Since the media era, information transmission is very rapid, so various unofficial news about the epidemic quickly spread, causing a certain amount of psychological pressure on ordinary people, especially medical staff [6]. Where A said, "I don't know why, I feel so scared! At that time, when I saw the news of a doctor's infection, I was so worried about that I was infected when I was at work"; C said, "I'm afraid that I will infect my family after infection, so when I get home, I would wash my whole body, and the washing machine is turned on four times a day"; E said, "Can we wear water shoes to work?" B said, "I used to have a good sleep, but now I haven't been able to fall asleep lately. I' $\mathrm{m}$ worried about that if I have some basic diseases, there would be an unthinkable consequence after infection, extreme nervousness and restlessness around me." D said, "I always feel something wrong with my lungs, is it possible for me to become an invisible infection because of the work? And I am worried about infecting others." H said, "I have to take public transport to work, so I am worried about the contact with NCP Infection.”

\subsection{The Work Pressures Increased Greatly in Epidemic Situation}

With the outbreak, the number of people diagnosed showed a straight upward trend [7]. When our hospital opened by January 27, all personnel entering the hospital was required to measure body temperature, and set up a special fever preview and triage point in the basis of the service desk as required. And the nurses at the outpatient service desk were in responsible. At first, the nurses used hand temperature gun to supervise the temperature of patients in our hospital, and the workload is a little heavy, so that although the infrared induction temperature measurement security door was urgently installed that day, the work of guiding and maintain order for the measurement was still overloaded. Early in the epidemic, our country is in short supply of protective materials. Among them, H said, "The hospital requires all patients to take the body temperature, the workload is in greatly increased and the close contact when taking their temperature makes us feel more pressure said, 'I feel too much pressure, and I can't sleep, need to have a rest, I will back to work when I feel better."

\subsection{The Contradictions and Hesitation During Triage in Epidemic Situations}

In the process of triage, in view of the complexity of the epidemic, such as the lack of specific medicine, the rapid development of the disease, the infection of a part of medical workers, and the nervousness of public, leading to many difficulties in preview and triage. The nurses at the outpatient service desk are worried about that the incorrect triage may cause others to become infected and their psychological guilt is increased. Among them, D said, "All the specialists have heard that they need to go to the fever clinic for investigation." B said, "yeah, there was a dialysis patient, yesterday's fever after dialysis has been treated locally but today, she fevers 
again, the local hospital asked her to go to the big hospital for nucleus acid assay. The patient has no history of contact and epidemiology, no respiratory symptoms, she called our hospital's specialist physician after coming hospital, were also asked to the fever clinic, because it would be in a big sin if she is a hidden infection [8] and go to the specialist to become a source of infection. But if she is not an NCP patient and then goes to the hot clinic, you know, the dialysis patient's resistance is low, if she gets infected with NCP as a result... In a word, my heart is also uneasy, it's really contradictory and tangled."E said, "There are still some patients that conceal their medical history, using the antipathetic instead of saying their fever out."

\subsection{Accept the Actuality of the Epidemic and Treat the Work Professionally}

After the implementation of comprehensive interventions, the mentality of nurses has changed positively. When in adjusting the shift schedule, D said, "Head nurse, if the work is understaffed, please arrange for me." The colleagues on vacation also said that they can come back to work in advance. They also accept the current situation of the epidemic of their psychological aspect and are familiar with the relevant knowledge of NCP, treat the work of preview and triage about fever with a professional attitude.

\section{Discussion and Conclusion}

The results of this survey show that, the nurses at the outpatient service desk are unfamiliar with the triage process and the lack of understanding of protection techniques, the lack of protective materials in the early stage of the epidemic, and heavy workload, causing many bad emotions such as worry, fear, nervousness and anxiety affected them, and they appeared obvious psychological stress response, In response to the cause of the nurses' bad emotions, we need to take corresponding countermeasures to alleviate their bad emotions.

\subsection{According to the Epidemic Situation, Formulate the Work Flow Scientifically and Guide the Nurses to Make Proper Triage}

The nurses at the service desk are responsible for the preview and triage of outpatients in hospitals and outpatients with fever. The head nurse of the outpatient services desk where the author work in responded quickly according to the current situation of the epidemic situation and the instructions in the superior documents, and formulated the work flow and relevant procedures of the preview and triage of fever. All personnel entering the outpatient hall are required to measure body temperature: (1) When the patient's body temperature is $<37.3^{\circ} \mathrm{C}$, guide to the corresponding specialist according to the symptoms and signs; (2) when the patient's body temperature is $\geq 37.3^{\circ} \mathrm{C}$, immediately transfer to the fever preview and triage point set up outside the gate, re-measure the body temperature and make an inquiry according to the
Registration Form of Preliminary Screening of Suspected Cases of novel corona virus infection, and then complete the registration. (3) patients that have a history of epidemiology and no symptoms should be asked to the fever clinic for screening; (4) patients with clear causes of fever should be directed to the corresponding specialist clinics, or to the ED if it is on holidays. (5) when the patient's body temperature $>37.3^{\circ} \mathrm{C}$, with epidemiological characteristics, in line with one of the concomitant symptoms or contact history in the Registration Form of Preliminary Screening, without any epidemiological characteristic, the diagnosis cannot be established and considered as a suspected case of new corona-virus infection, start immediately; (1) Distribute the medical surgical masks to the patients and guide them how to wear; (2) Lead the patients to hot clinics from outside the main entrance of the hospital and assist them to register by themselves; be responsible for leading patients to hot clinics for CT examinations and other work. And the team will also provide a basis of formulating a more scientific and rigorous epidemic prevention process based on positive feedback and suggestions from daily work. Smooth work flows can effectively reduce the pressure in the nurses and improve work efficiency. The establishment of the triage works flow in the epidemic situation provided scientific guidance of the nurse triage, thus alleviated the anxiety of them.

\subsection{Strengthen the Relevant Theoretical Knowledge and the Safeguard Skills Training as Nurses of Covid-19, and Do A Good Job of Personal Protection}

The head nurse of office organized the service desk nurses to learn the latest knowledge and related documents of the NCP online and offline and the conducted online assessments. According to the documents of the Department of infection and Control of the hospital, the preview and triage (the service desk) is the 1-level prevention and control: (1) wear disposable working caps, disposable medical surgical masks and white lab coats, disposable isolation gowns, and disposable latex gloves if necessary; (2) clean hands before wearing masks or after removing them; (3) perform personal hygiene before work and pay attention to the protection against the respiratory tract and mucous membranes; (4) strictly abide by the principle of standard prevention; (5) strictly abide by the rules and regulations of disinfection and isolation; (6) execute strictly the system about hands washing and hands disinfecting. And on this basis, the preview and triage point of fever will equip N95 masks, protective clothing, and shoe covers. All nursing staff must receive training in proper wearing of protective equipment and preventive measures [9]. During the work, it is necessary of mutual supervision between nurses about the respective protective measures to ensure that they are properly in place and that they are in place to protect everyone's life in safe.

\subsection{Communicate and Coordinate Up and Down to Ensure the Protection Against Protective Materials}

In the early stage of the NCP epidemic, there was a 
nationwide shortage of protective materials. Our hospital issued uniformly protective materials to the working nurses in accordance with the stipulations of the infection and control documents of the hospital Grading Protection Requirements of Medical Staff and Standard Operating Rules for the Use of Medical Protective Masks; The department should arrange for permanent stuff to get protective materials on time every day and do a good job of registration and filing for each use. In case of shortage of supplies, the department leaders should try to communicate and coordinate with the equipment department, nursing department and medical department to ensure that the protective supplies are in place, ensure the supply of protective materials of the nurses, and ease the nurses mood about the anxiety and nervous caused by their lack of protective materials.

On February 23,2020, the National Personnel Department's Notice on the Central Leading Group's Response to NCP Infection Outbreak about the all-round Implementation of a number of measures to Further Protect and Care for Medical Staff [10] Pointed out that it is necessary to promptly and effectively do psychological adjustment and counseling of medical staff. The head nurse of the department should timely and actively ask for the nurses' psychological changes and their rest, conduct reasonable scheduling and group psychological intervention online [11]; Encourage nurses to communicate with each other to express their own experiences and feelings and soothe each other, and also encourage the expression of their negative emotions, which is to the benefit of their mental health of nursing staff under the epidemic [12]; Concerned about the physical and mental status of the nurses and the health status of family members, daily monitor their temperature and clock at their health status. Timely psychological intervention according to the psychological status of the nurses at the service desk, and organize all members to watch the online lectures what is about the sleep adjustment and the common psychosomatic reactions and cop strategies of the medical staff in our hospital under the outbreak. Encourage the family to carry out five health exercises, eight centigrams boxing, Thai chi Chan, etc, enjoy soothing music, cook some food, exercise at home, enrich the day off, maintain a good mood and good sleep.

\section{References}

[1] F. t. C. N. C. Investigating, R. Team. A Novel Coronavirus from Patients with Pneumonia in China, 2019.

[2] State Council of the People's Republic of China. Emergency Regulations for Public Health Emergencies. Occupational Health and Emergency Rescue (02) (2003) 24-27.

[3] Feng, Shengyao. Theory and Application of Focus Interviews. Modern Education Series (4) 20-23, 65.

[4] Li, Zheng. Research Methods in Nursing. 2012.

[5] Zhang, Song. Zhang, Deshan. The Nature and Correction of Anxiety. Journal of Xuchang University (1) (1998) 126-127.

[6] Zhang, Yi. Investigation of Psychological Status of Nurses During SARS Period. Chinese Journal of Modern Nursing 010 (6) 497-499.

[7] The latest situation of the new coronavirus pneumonia epidemic situation. pdf.

[8] Wang, Ping. Analysis of the prevention and treatment efficacy of 182 cases of latent infection in the epidemic of human adenovirus type 7 epidemic. Chinese Journal of Pulmonary Diseases (electronic version) (2018).

[9] Cai, Lidong. Safety Application of New Coronavirus Pneumonia Antiviral Drugs. Journal of Adverse Drug Reactions 22 (00) (2020) E001-E001.

[10] Deng, Rong. Chen, Fang. Liu, Shanshan. Yuan, Li. Song, Jinping. Factors influencing the psychological stress of medical staff in the new coronavirus pneumonia isolation ward. China Infection Control Journal (2020).

[11] Ma, Ning. Ma, Hong. Li, Lingjiang. Expert analysis of "Emergency Psychological Crisis Intervention Guidelines for New Coronavirus Infected Pneumonia Outbreak". Chinese Psychiatric Journal (2020).

[12] Xu, Jiali. The effect of collective intervention of focus solution model on psychological capital of operating room nurses. Qilu Nursing Journal (16) 33-36. 